\title{
Nefrectomía parcial laparoscópica transperitoneal en el tratamiento del tumor renal
}

\author{
Rosales Bordes A, Salvador Bayarri J, de Graeve N, Palou Redorta J, Villavicencio Mavrich H.
}

Servicio de Urología. Fundació Puigvert. Barcelona.

Actas Urol Esp 2006; 30 (5): 492-500

\section{RESUMEN}

\section{NEFRECTOMÍA PARCIAL LAPAROSCÓPICA TRANSPERITONEAL EN TRATAMIENTO DEL TUMOR} RENAL

La cirugía laparoscópica constituye una técnica quirúrgica que el urólogo debe incorporar a su armamentario quirúrgico. Su realización intenta simular los pasos quirúrgicos de la cirugía abierta, así como sus indicaciones. La nefrectomía parcial laparoscópica es una técnica compleja que implica para su desarrollo una experiencia amplia en el manejo de las técnicas endoscópicas. Presentamos nuestra experiencia de 35 nefrectomías parciales laparoscópicas transperitoneales con una media de seguimiento de 25 meses. El tiempo medio de cirugía fue de 200 minutos, el sangrado de 190 cc, y la estancia media de cinco días. Se han objetivado dos sangrados postoperatorios, ninguna conversión y en dos casos se informó de márgenes positivos, optándose por una actitud conservadora.

Palabras clave: Nefrectomía parcial laparoscópica. Tumor renal. Técnica quirúrgica.

\section{ABSTRACT}

TRANSPERITONEAL LAPAROSCOPIC PARTIAL NEPHRECTOMY IN THE RENAL TUMOR TREATMENT

Laparoscopic surgery is a surgical technique the urologist should add to his surgical armamentarium. Its performance tries to mimic the surgical phases of open surgery, and also its indications. Laparoscopic partial nephrectomy is a sophisticated technique that requires wide experience in the performance of endoscopic strategies. We are submitting our experience with 35 laparoscopic partial transperitoneal nephrectomies with a mean follow-up of 25 months. The mean surgical time was 200 minutes, the mean bleeding $190 \mathrm{cc}$, and the mean hospitalisation five days. Two postoperative bleedings were identified, there were no conversions, and positive margins were notified in two cases, whereupon a conservative attitude was adopted.

Key words: Laparoscopic partial nephrectomy. Renal tumour. Surgical technique.

$\mathrm{E}^{\prime}$ diagnóstico incidental del cáncer renal ha aumentado en la última década gracias al uso de la ecografía y de las demás técnicas de imagen para estudio de la cavidad abdominal ${ }^{1}$. Desde que Clayman ${ }^{2}$ en 1991 describió la primera nefrectomía laparoscópica para el tratamiento del tumor renal, esta técnica se desarrolló rapidamente permitiendo que Winfield ${ }^{3}$ en 1993 practicara la primera nefrectomía parcial laparoscópica. Los resultados obtenidos por Gill ${ }^{4}$, Guillonneau $^{5}$, Janetschek ${ }^{6}$ y Rassweiler ${ }^{7}$, muestran la reproductibilad, eficacia y seguridad de la técnica cuando es realizada correctamente.

Se ha demostrado que la nefrectomía parcial presenta unos resultados similares en cuanto a supervivencia comparado con la nefrectomía 
radical en pacientes seleccionados, aunque esta técnica es difícil, siendo el control de la hemostasia y el cierre de la vía urinaria los dos puntos más conflictivos. Un estudio de Gill, comparando la cirugía parcial laparoscópica y la cirugía abierta demostró que aquella presenta un mayor periodo de isquemia caliente, con más complicaciones intraoperatorias, aunque la función renal fue preservada por igual y los pacientes se recuperaron más rápidamente ${ }^{4}$.

En muchos casos se requiere la hipotermia del riñón para poder realizar dicha técnica por el apremio que representa la limitación en el tiempo a que se ve sometido el cirujano para llevarla a cabo en menos de 30 minutos. En los últimos años la adopción de la cirugía laparoscópica como otra técnica más dentro del armamentario quirúrgico que el urólogo debe ofrecer a sus pacientes, y la comprobación que su eficacia oncológica es similar a la cirugía abierta, ha motivado que la nefrectomía laparoscópica sea el tratamiento de elección en la mayor de los tumores renales. Sin embargo la dificultad que conlleva la práctica de la nefrectomía parcial laparoscópica ha impedido que se haya extendido su uso, pues requiere de un cierto dominio de la sutura intracorpórea y de la disección endocavitaria. Este hecho ha producido que se hayan utilizado distintas técnicas no exeréticas para poder tratar los pequeños tumores renales, como la crioterapia o la radiofrecuencia que presentan una media de recurrencia tumoral del $8 \% 8$. Con el fin de obtener una mejor hemostasia, han aparecido distintos productos biológicos con propiedades hemostáticas y sellantes para disminuir la necesidad en el uso de sutura intracorpórea. La técnica laparoscópica intenta reproducir los mismos pasos de la cirugía abierta, generalmente mediante control del pedículo vascular renal, manteniendo dos prioridades básicas: control oncológico con mínima pérdida nefronal e intervención con la menor morbilidad posible ${ }^{9}$.

\section{MATERIAL Y MÉTODOS}

Desde febrero de 2002 a abril de 2005 hemos realizado en nuestro centro 35 nefrectomías parciales laparoscópicas por tumor renal; el abordaje fue transperitomeal, siendo 25 varones y 10 mujeres con una edad media de 64 años (26-77). Todas las cirugias fueron realizadas mediante técnica laparoscópica pura a excepción de un caso el cual se finalizó mediante técnica mano asistida. En 19 casos la nefrectomía parciales laparoscópicas correspondió al lado derecho, siendo 26 izquierdas. La media de tamaño de las masas renales resultó 2,6 cms. (1,5-7). Dos cirugías se realizaron sobre pacientes monorrenos. En todos los casos los tumores fueron masas exofiticas renales a excepción de dos casos en que no lo eran y al no deformar la superficie renal se localizaron mediante ecografía intracorpórea con una sonda de alta frecuencia.

Se posiciona a los pacientes en decúbito lateral con un ángulo de $30^{\circ}$ (Fig. 1). Se les coloca un vendaje neumático secuencial en extremidades inferiores, sonda vesical y nasogástrica y se les administra un gramo de cefonicid ev. como profilaxis antibiótico. El catéter gástrico se retira en el momento de finalizar la intervención.

La colocación del primer trócar se realiza tras una incisión de laparotomía de $2 \mathrm{~cm}$ a nivel del borde externo de los músculos rectos anteriores del abdomen, por donde se introduce un trócar de $12 \mathrm{~mm}$ a través del cual colocaremos una óptica de $0^{\circ}$.

Generalmente hemos utilizado 5 trócares en el abordaje del lado izquierdo y 5 ó 6 en los tumores del lado derecho (Fig. 2).

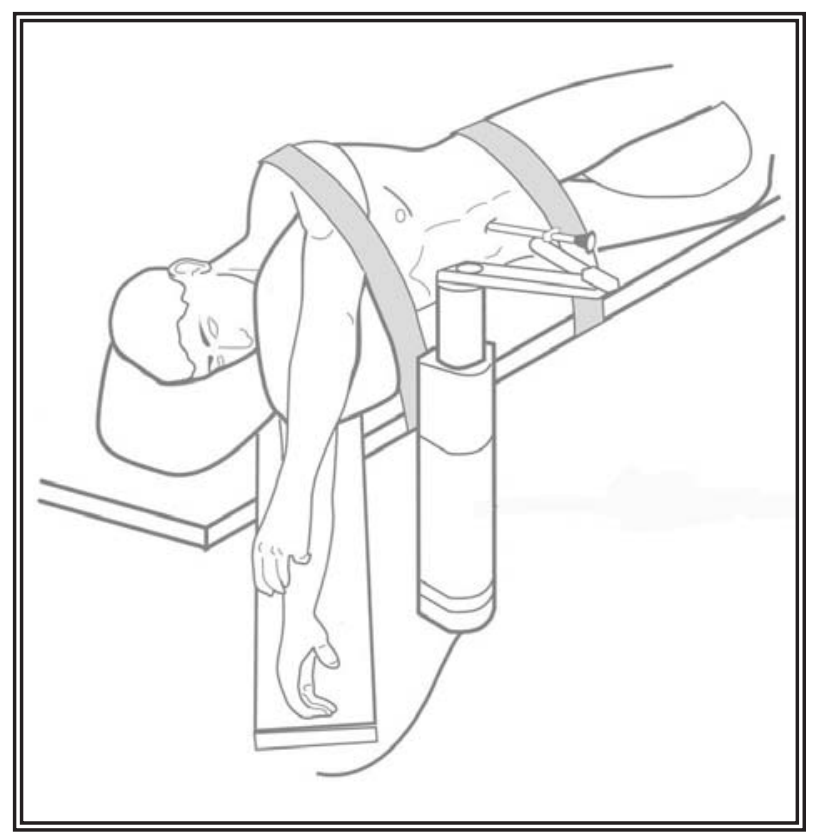

FIGURA 1 


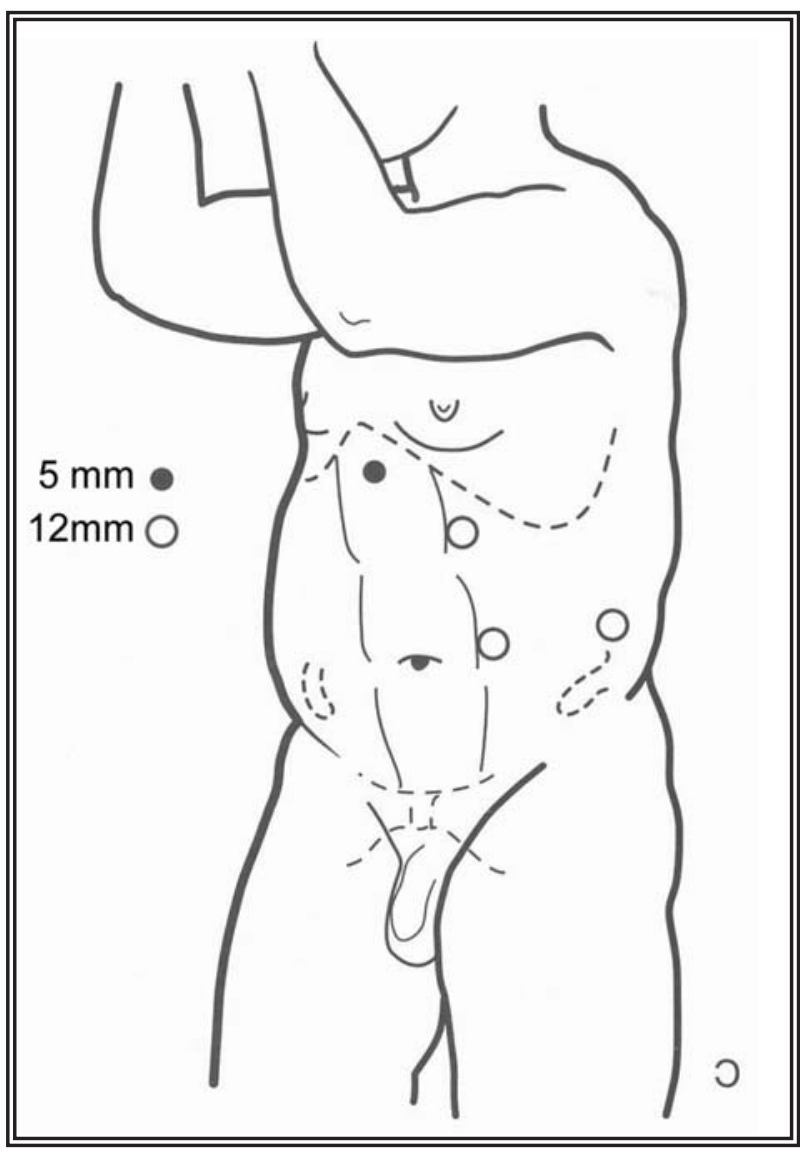

FIGURA 2

La sistemática de la técnica quirúrgica se realiza de forma reglada. Tras una amplia decolación se realiza una maniobra de Kocher en el lado derecho, y seguidamente una disección de hilio renal con la colocación de un torniquete de Rumel a nivel de la arteria o arterias renales (Fig. 3).

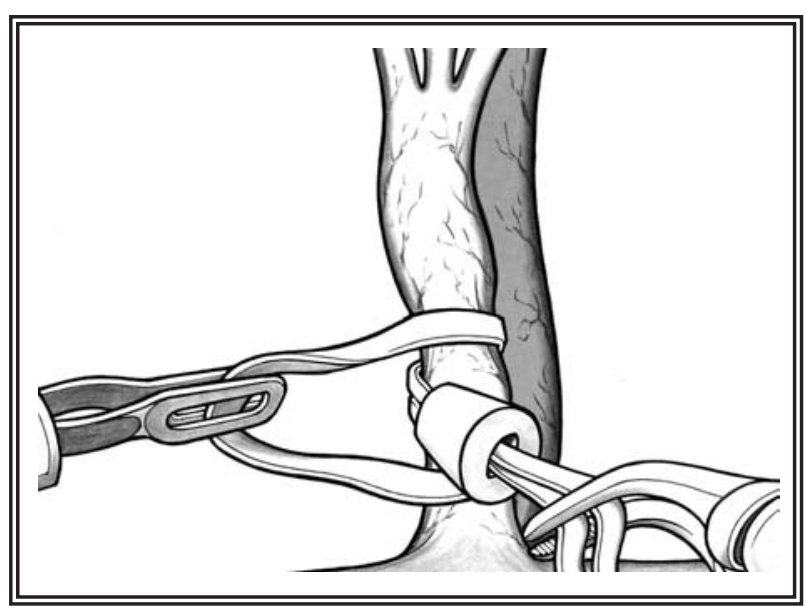

FIGURA 3
En un caso se colocó un clamp de Satisky laparoscópico a través de un trocar flexible plástico, correspondiendo un caso de una heminefrectomía. Previo al clampado vascular se administra $20 \mathrm{mg}$ de manitol por vía endovenosa y se realiza la oclusión arterial 3 minutos después de su perfusión, para proteger la función renal de la isquemia caliente.

En 5 casos se realizó la exéresis tumoral sin control del pedículo. La disección de la superficie renal una vez liberada de su grasa, permite una buena inspección renal y la posibilidad de una óptima movilización renal, lo que nos facilitará el procedimiento. Una vez localizado el tumor realizamos una ecografía endocavitaria con un transductor de alta resolución en 16 casos, su uso se justificó para la localización del tumor o cuando existían dudas sobre la profundidad de la lesión y su relación con la medular renal. Delimitaremos el borde de sección (con margen de seguridad) con diatermia monopolar. Un detalle relevante es la posibilidad de cambio de la óptica a través de otro puerto de $10 \mathrm{~mm}$. Dependiendo de la localización del tumor para conseguir una visión y un ángulo de trabajo óptimo.

Normalmente ocluimos la arteria renal mediante torniquete de Rumel modificado para la cirugía laparoscópica. Se practica la nefrectomía parcial o la tumorectomía mediante corte monopolar en la corteza renal y mediante corte frío en la medular, coagulando selectivamente los pequeños vasos arteriales que aparezcan durante la sección (Fig. 4). Tras ello se realiza la sutura de la vía cuando ésta ha sido abierta de forma extensa mediante sutura con monocryl del 3/0. Se colocará trombina bovina (Floseal) y Surgicel enrollado en el lecho del tumor y puntos de colchonero con Vicryl del 0 o puntos de sutura mediante clips plásticos apropiados en caso que dicha sutura sea necesaria. Tras afrontar los márgenes se coloca un adhesivo biológico (Bioglue) compuesto por albúmina y glutaraldehido. En 7 ocasiones realizamos la coagulación del lecho con bisturí de Argón. Tras comprobar la correcta hemostasia se colocó un drenaje aspirativo y cerramos los orificios de los trócares de $12 \mathrm{~mm}$. La extracción de la pieza quirúrgica es practicada mediante endocath de $10 \mathrm{~mm}$. 


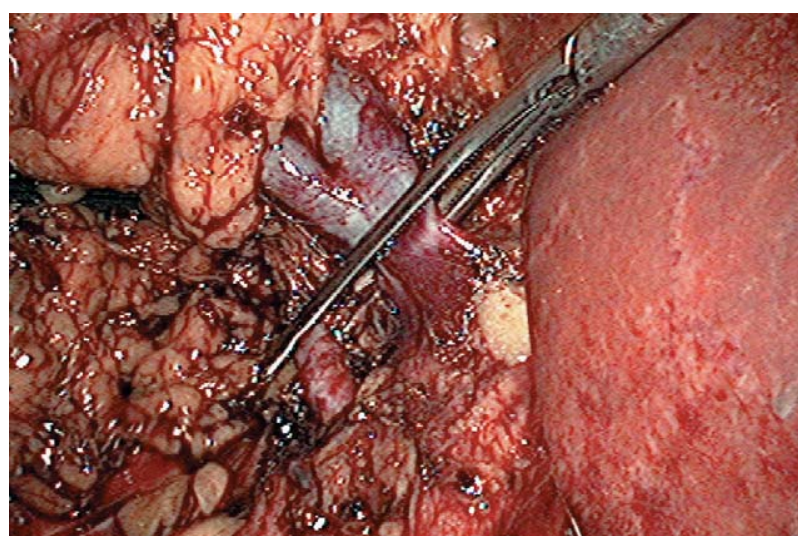

FIGURA 4

\section{RESULTADOS}

El tiempo medio de la intervención fue de 200 minutos, el sangrado de 190 cc. (50-400), la estancia media de 5 días (4-7). Realizamos sutura específica de la vía urinaria en 4 casos, no apareciendo fístulas urinarias. El tiempo de isquemia caliente resultó de 28 minutos (20-43) El estudio histopatológico demostró 29 carcinomas renales, 3 angiomiolipomas, 2 oncocitomas y un nefroma quístico. Todos fueron $\mathrm{T} 1$ excepto un tumor de 7 $\mathrm{cm}$ (T2), en el que realizamos una heminefrectomía. En dos casos se objetivaron márgenes positivos, uno en un carcinoma renal y en un caso de oncocitoma. En los dos casos se usó el bisturí de Argón para la coagulación superficial del lecho y tras 25 meses de seguimiento no se han apreciado recidivas tumorales. Como complicaciones hemos tenido 2 sangrados postoperatorios sintomáticos que requirieron transfusión y se manejaron de forma conservadora.

Tras un tiempo medio de seguimiento de 24 meses, no se han evidenciado recidivas tumorales y todos los pacientes se hallan vivos. El seguimiento de los pacientes se ha realizado mediante ecografia semestral y TAC anual, así como con determinación de función renal. En un caso de una paciente monorrena, tras la cirugía laparoscópica, presentó un aclaramiento de creatinina de $25 \mathrm{ml} / \mathrm{m}$.

\section{DISCUSIÓN}

La nefrectomía parcial laparoscópica intenta simular los pasos quirúrgicos de la cirugía abierta, así como sus indicaciones; en la actualidad se acepta como adecuada en pacientes monorrenos, con tumor renal bilateral y en enfermos con insuficiencia renal (indicaciones imperativas) y en aquellos con tumores renales inferiores o igual a $4 \mathrm{~cm}$ de diámetro, exofíticos con riñón contralateral normal (indicaciones electivas o programada). El abordaje de tumores mayores está en discusión por el riesgo de recurrencia tumoral, multifocalidad o dificultad técnica, aunque el aumento de la experiencia del cirujano plantea modificar el límite y elevarlo con ello hasta $7 \mathrm{~cm}^{9}$. Generalmente se desestiman para el abordaje parcial laparoscópico los tumores de localización medial o cercanos al seno renal por el riesgo de apertura de la vía urinaria, aumento de sangrado postoperatorio, posibilidad de crear áreas necróticas de parénquima renal o riesgo de incisión de la pseudocápsula del tumor renal comprometiendo por todo ello el resultado oncológico del procedimiento. Aunque la experiencia de los distintos grupos favorece que se amplien las indicaciones de este tipo de técnica aumentado el riesgo de complicaciones.

El abordaje transperitoneal de los tumores anteriores y situados en el polo inferior es más sencillo que los que se hallan en la cara posterior $\mathrm{y}$ superior, los cuales serían unos candidatos ideales para un abordaje retroperitoneal, aunque también una completa disección del riñón puede hacer posible una completa exposición renal en un abordaje transperitoneal ${ }^{9}$.

La cirugía mano asistida puede ser útil en casos concretos de tumores de gran volumen, pudiendo controlar mejor la hemorragia con la compresión manual y alargando así el tiempo de trabajo, minimizando por ende el periodo de isquemia caliente en casos de difícil acceso o de gran volumen ${ }^{10}$.

Los antecedentes de cirugías previas pueden a su vez constituir una dificultad añadida en el abordaje anterior, por la posibilidad de encontrar adherencias que dificulten la disección renal, pero una realización de minilaparotomía o la colocación de trócares bajo visión, así como una disección cuidadosa, pueden hacer posible este procedimiento. La obesidad, aunque en un principio se demostró que aumentaba la morbilidad de esta técnica, hoy en día ya existen experiencias que demuestran que éstos pacientes se benefician de abordajes menos agresivos ${ }^{11}$. 
El uso de drenaje del sistema colector mediante un cateterismo ureteral en este tipo de intervenciones está en discusión. Autores como Gill ${ }^{9}$ y Bermude ${ }^{12}$ lo usan para evaluar la dimensión de la apertura del sistema colector mediante la instilación de índigo carmín. Bove ${ }^{13}$ refiere que en su experiencia no existe diferencia entre colocar o no un catéter ureteral para minimizar las fístulas, aunque su serie es limitada. Al igual que Johnston ${ }^{14}$, nosotros no lo utilizamos de forma rutinaria y aunque hemos necesitado suturar en pocos casos la vía urinaria, no hemos tenido fugas. En el estudio referido, de 100 casos intervenidos, en los primeros 75 procedimientos apareció 1 fístula, ello sin cerrar nunca la vía urinaria ya que las lesiones eran periféricas y los casos escogidos. Creemos que la compresión del parénquima mediante los puntos hemostáticos reduce la superficie de vía urinaria abierta y minimiza la posibilidad de fístula. Sólo en caso de lesiones extensas, que obliguen a realizar una heminefrectomía (resección de $>30 \%$ del parénquima renal) cuando preveamos que la apertura de la vía será extensa o que una hemostasia sea previsiblemente difícil lo que conlleve al aumento de presión de la vía urinaria por la ocupación de coágulos, en esos casos creemos que sería realmente efectivo el uso de un catéter ureteral de drenaje.

Se han utilizado diversas formas para realizar la nefrectomía parcial laparoscópica: sin oclusión del pedículo, mediante oclusión hiliar en isquemia caliente y mediante isquemia fría.

La disección del pedículo vascular renal durante la nefrectomía parcial laparoscópica puede ser una maniobra algo difícil para cirujanos sin mucha experiencia en cirugía laparoscópica renal, y por lo tanto puede consumir mucho tiempo y llevar implícito un riesgo de lesión de los vasos. Por esto se han descrito distintos mecanismos para practicar la exéresis tumoral sin clampado arterial. También la mejora en los distintos agentes hemostáticos biológicos ha favorecido que se puedan abordar dichos procedimientos sin disección hiliar. Se han utilizado distintas fuentes de energía como la corriente mono y bipolar (ligasure), ultrasonidos (ultracision y Cusa), radiofrecuencia, suero salino a presión (Hidrojet), microondas, y láser (Holmium, KTP) ${ }^{8}$. Tendrían como ventaja obviar la necesidad de disección del pedículo renal, ganando tiempo, y la ausencia de isquemia renal. Como inconvenientes serían la posibilidad de un mayor sangrado y que al disminuir la buena visibilidad para realizar una resección por el plano correcto de la medular, podrían favorecer los márgenes positivos.

Otra posibilidad consiste en no ocluir el pedículo en caso de tumores periféricos, pudiéndose realizar una compresión del parénquima renal, ya sea mediante la introducción de la mano o mediante cinchas compresivas, que introducidas por los trócares y rodeando uno de los polos renales, provocaran una isquemia selectiva que permitiese realizar la exéresis del tumor. La utilización de dichos dispositivos no han sido muy reproducibles y por ello pocos autores los utilizan; en cambio el recurso de colocar un dispositivo "mano asistido" puede alargar el tiempo de trabajo, a costa de una posición más incómoda, pero que permita al fin una correcta realización de la técnica ${ }^{8}$.

Distintos estudios experimentales han relacionado la duración de la isquemia caliente con la alteración de la funcionalidad renal. Tras una isquemia de 30 minutos la recuperación de la función renal es total tras varias horas, una isquemia de 60 minutos provoca en la rata una disfunción renal con una necrosis tubular que se puede recuperar en 30 dias y una isquemia caliente de 90 minutos provoca una pérdida de unidad renal en el $83 \%$ de los casos $^{15}$.

Shekarriz ${ }^{16}$ realizaron gammagrafias renales tras la práctica de nefrectomías parciales laparoscópicas con una media de isquemia caliente de 22,5 minutos con un rango entre 10 y 40. En ningún caso se apreció pérdida de ninguna unidad renal. Kane ${ }^{17}$ también apoya esta hipótesis afirmando que tras 15 procedimientos con un tiempo de isquemia de 43 minutos la función renal global no se vio afectada. Ello implica que los métodos de nefrectomía parcial laparoscópica que utilicen isquemia caliente requieren que ésta no sobrepase los 30-40 minutos para salvaguardar la función renal.

Para la oclusión de los vasos renales se han utilizado distintos métodos, siendo los más utilizados: los bulldocks, las pinzas de Satinsky laparoscópico y el torniquete de Rumel modificado en 
nuestro centro para la cirugía laparoscópica ${ }^{18}$. Todos son buenos dispositivos pero requieren de una serie de detalles en cuanto a su uso. Los clamps vasculares se colocan mediante unas pinzas que "abrazan" a los bulldocks en su recorrido por la cavidad abdominal. Es posible que al abrirlos para colocarlos en los vasos o para extraerlos una vez finalizado el procedimiento, puedan desprenderse y caer dentro del abdomen, siendo a veces difícil su rescate. La pinza de Satinsky es útil pues puede evitar una disección exhaustiva del pedículo vascular y puede realizar una oclusión total de arteria y vena, pero su colocación debe ser muy delicada ya que en ocasiones pueden producirse desgarros venosos o lesiones en la intima de arterias renales con severa aterosclerosis. A su vez el vaseloop del torniquete de Rumel puede seccionarse si se utilizan clips metálicos, hecho que se minimiza si se utilizan clips plásticos como los de la casa Weck (Hem-o-lock).

Realizar una isquemia fría correcta, alcanzando los $20 \mathrm{C}^{\circ}$ en la corteza renal, puede ser una empresa difícil en la cirugía laparoscópica. Diversos autores han diseñado distintas formas para intentar disminuir la temperatura renal y así aumentar el tiempo de trabajo para realizar la nefrectomía parcial con el mínimo daño renal. Janetscheck ${ }^{6}$ describe una técnica de isquemia fría mediante la introducción de una cánula intraarterial con la perfusión a nivel del ostium de la arteria renal de una solución de Ringer lactato a $4 \mathrm{C}^{\mathrm{o}}$, ocluyendo la arteria mediante un catéter balón. Este método es poco reproducible y el autor sólo lo emplea en casos muy seleccionados de tumores grandes. Landman ${ }^{19}$ enfría el riñón mediante la instilación retrógrada de solución salina fría a través de un catéter ureteral, pero no consigue una temperatura de enfriamiento óptima. Gill ${ }^{20}$ y otros autores ${ }^{21}$ describen distintas formas de introducir hielo "pillé" a través del orificio de un trócar, tras colocar el riñón en el interior de una bolsa, donde poder enfriarlo sin que se pierda el hielo por la cavidad abdominal. Sin embargo ninguno de estos métodos ha sido ampliamente aceptado y los mismos autores en su medio lo utilizan en casos muy seleccionados.

Otro punto importante es que para la práctica de la nefrectomía parcial laparoscópica es importante el poder contar con una sonda ultrasónica intracorpórea de alta frecuencia para poder realizar una definición más precisa del tamaño, localización y relación del tumor con las estructuras más profundas (vasos y vía urinaria). Puede distinguir de forma precisa la ocupación de un quiste complicado y definir la orientación del inicio de la sección del parénquima renal. Para una aplicación mejor la sonda debe colocarse en la cara opuesta donde se halla la lesión para que la zona problema se aleje de la superficie del ecógrafo mejorando con ello la definición ${ }^{22}$.

Como hemos comentado uno de los puntos críticos de este tipo de técnica es conseguir una adecuada hemostasia tras la resección tumoral. Para ello se han descrito el uso de distintos materiales y distintas fuentes de energía ${ }^{8}$. En conclusión podríamos decir que la utilización de métodos sin oclusión vascular estarian indicados en tumores pequeños $(<3 \mathrm{~cm})$, exofíticos y con poca penetración parenquimatosa $(<10 \mathrm{~mm})$. En esos casos todas las fuentes de energía pueden ser válidas y eficaces. Para lesiones mayores y más profundas es indispensable la oclusión del pedículo vascular renal y la hemostasia deberá incluir además de agentes biológicos hemostáticos, la colocación de sutura mediante puntos de "colchonero" clásicos o una plicatura del parénquima renal mediante colocación de sutura sujeta con clips plásticos (Lapra-Ty o Hem-o-lock).

Existen varios agentes hemostáticos y sellantes en el mercado que nos pueden ayudar a realizar una hemostasia correcta en esta técnica. Actualmente contamos en nuestro medio con cuatro sustancias hemostáticas con características diferentes. Contamos con el Floseal que está formado por gránulos de trombina bovina; esta sustancia desencadena en contacto con la sangre el proceso de la cascada de la coagulación a nivel local. Requiere para su eficacia que se mantenga una presión sobre la zona cruenta que generalmente se consigue mediante la interposición de un rollo de celulosa (surgicel). El Floseal es de fácil almacenamiento y su aplicación inmediata requiere una preparación previa minutos antes de su aplicación. Su alto poder de autocompactación implica la necesidad de disponer de un aplicador metálico, poco adherente y el limpiado del interior del mismo cada vez que se utilice. El Tyssucol está compuesto por adhesivo de fibrina 
que requiere ser almacenado a baja temperatura y preparado 30 minutos antes de su aplicación. Una vez desenfriado no puede ser reutilizado. Su aplicación se realiza mediante una cánula plástica. El Bioglue está constituido por una mezcla de albúmina y glutaraldehido. Su aplicación es inmediata mediante cánula plástica, donde se mezclan en el momento de su aplicación sus componentes. El producto es una sustancia de aspecto y consistencia plástica traslúcida que se adhiere rápidamente a los tejidos donde es aplicado. En la Universidad de Michigan ${ }^{14}$ se realizó un estudio prospectivo sobre la eficacia de los distintos sellantes biológicos en nefrectomías parciales en cerdos. La conclusión fue que la mayoria de los agentes hemostáticos consiguen alguna hemostasia, siendo el Floseal y el Tyssucol los de mejor resultado, aunque en resecciones parenquimatosas importantes o apertura de la vía urinaria, sólo el refuerzo mediante algún tipo de sutura fue realmente efectivo.

Al finalizar la cirugía es recomendable dejar un drenaje, aspirativo o no, pero no en contacto directo con la zona cruenta renal. Solemos colo- car un drenaje de Redón del 10 ch, retirando la aspiración a las 24 horas si el débito es menor de 50 cc al día, y retirándolo a las 48 horas si éste sigue siendo no productivo. Aparte de la técnica quirúrgica hay que considerar la necesidad de mantener la presión sistólica < $160 \mathrm{~mm}$ de $\mathrm{Hg}$, usando hidralacina $\mathrm{u}$ otro hipotensor según necesidad, disminuyendo con ello el riesgo de hemorragias postquirúrgica ${ }^{14}$.

Mediante una buena técnica quirúrgica, tras adecuada elección del caso escogido y con el uso de distintas técnicas de apoyo peroperatorias como los ultrasonidos, se puede disminuir la recurrencia del tumor en el remanente renal, tanto por escisión incompleta como por la aparición de un tumor multifocal. La biopsia intraoperatoria en esta técnica, según nuestra opinión no debe de ser una práctica rutinaria, como sostiene Kubinski ${ }^{23}$. Este autor obtuvo un margen positivo en el 1,3\% de sus nefrectomías parciales laparoscópicas, apareciendo en su serie de recidiva local en un tumor T3, con biopsia del lecho de resección negativa. En la revisión bibliográfica los márgenes positivos oscilan entre el 0 y el 8\%, siendo la media de 1,8\% (Tabla 1). Aunque existe

Tabla 1

Experiencias seleccionadas de nefrectomía parcial laparoscópica por tumor renal

\begin{tabular}{|c|c|c|c|c|c|c|c|}
\hline & Pacientes (n) & Ruta & $\begin{array}{c}\text { Tamaño } \\
\text { medio del } \\
\text { tumor }(\mathrm{cm})\end{array}$ & $\begin{array}{c}\text { Hemorragia } \\
\text { (\%) }\end{array}$ & $\begin{array}{c}\text { Fuga de } \\
\text { orina } \\
(\%)\end{array}$ & $\begin{array}{c}\text { Margen } \\
\text { positivo } \\
\text { final (\%) }\end{array}$ & $\begin{array}{c}\text { Seguimiento } \\
\text { medio } \\
\text { (meses) }\end{array}$ \\
\hline Rassweiler et al. & 53 & TP/RP LS & 2,3 & $5(9 \%)$ & $5(9 \%)$ & NA & 24 \\
\hline Stifelman et al. & 11 & TP LAM & 1,9 & $0(0 \%)$ & $1(9 \%)$ & $0(0 \%)$ & 8 \\
\hline Gettman et al. & 10 & TP/RP LS & 2,1 & $0(0 \%)$ & $0(0 \%)$ & $0(0 \%)$ & NA \\
\hline Jeschke et al. & 51 & TP/RP LS & 2 & $1(2 \%)$ & $3(6 \%)$ & $0(0 \%)$ & 34 \\
\hline Guillonneau et al. & 28 & TP LS & 2,2 & $5(18 \%)$ & $0(0 \%)$ & $0(0 \%)$ & 5,9 \\
\hline Richter et al. & 10 & TP LS & 2,7 & $0(0 \%)$ & $0(0 \%)$ & $0(0 \%)$ & NA \\
\hline Simon et al. & 20 & TP LS & 2,1 & $1(5 \%)$ & $0(0 \%)$ & $0(0 \%)$ & 8,2 \\
\hline Gill et al. & 100 & TP/RP LS & 2,8 & $6(6 \%)$ & $3(3 \%)$ & $3(3 \%)$ & 13 \\
\hline Kim et al. & 79 & TP LS & 2,5 & $4(5 \%)$ & $2(3 \%)$ & $2(3 \%)$ & 20 \\
\hline Janetschek et al. & 15 & TP LS & NA & $2(13 \%)$ & $0(0 \%)$ & $0(0 \%)$ & NA \\
\hline Brown et al. & 30 & TP LAM & 2,6 & $6(20 \%)$ & $6(20 \%)$ & $0(0 \%)$ & 8,8 \\
\hline Pruthi et al. & 15 & TP LAM & 2,7 & $0(0 \%)$ & $0(0 \%)$ & $0(0 \%)$ & NA \\
\hline Kane et al. & 27 & TP LS & 2,6 & $0(0 \%)$ & $2(7 \%)$ & $1(4 \%)$ & NA \\
\hline Serie actual & 100 & TP/RP LS/LAM & 2,5 & $9(9 \%)$ & $2(2 \%)$ & $3(3 \%)$ & 15 \\
\hline F. Puigvert & 35 & TP LS & 2,6 & $2(8 \%)$ & $0(0 \%)$ & $2(8 \%)$ & 25 \\
\hline Total & 584 & & 2,5 & $7,1 \%$ & $4,4 \%$ & $1,8 \%$ & 14 \\
\hline
\end{tabular}


esta cifra de márgenes positivos, ello no se traduce en la necesaria aparición de recurrencia local. La valoración del margen por el patólogo se realiza mediante tinción en fresco con tinta china, lo que muchos de los márgenes positivos pueden ser debidos a una mala manipulación quirúrgica de la pieza de tumorectomía renal. Hay que sumar además el tiempo necesario para obtener el dictamen, con lo que se alarga el tiempo de isquemia renal. El hecho de que la mayoría de los tumores renales intervenidos mediante esta técnica son $\mathrm{T} 1$, hace poco probable que los márgenes sean positivos si la técnica quirúrgica es correcta. El uso complementario de coagulación con bisturí de Argón, el cual produce una coagulación superficial sobre el lecho quirúrgico, puede disminuir aún más la posibilidad de una recurrencia $^{14}$. Las biopsias del lecho quirúrgico sólo estarian indicadas según nuestra opinión, sólo en casos en que el examen macroscópico mostrara una resección de la pieza inadecuada.

Las complicaciones más frecuentes en este tipo de técnica son la hemorragia peroperatoria que se aproxima al $7 \%$ ( 2 y el $20 \%$ ) y la fístula urinaria en un $4,5 \%$ de los casos (3-20\%). Otras complicaciones menos frecuentes que se han descrito son hipercapnia, embolismo gaseoso, así como lesiones intestinales, esplénicas o íleo prolongado.

Tras la valoración de tres años de seguimiento de varias series, parece que esta técnica presenta unos resultados semejantes a los obtenidos mediante cirugía abierta. De todas maneras cabe esperar los resultados a más largo plazo para ver que la evolución sea comparable entre ambas técnicas. En un artículo de la Cleveland Clinic $^{24}$ la tasa de recurrencia total fue del $4 \%$ siendo del $0 \%$ para los pT1, del $2 \%$ para los pT2 siendo del $8 \%$ para los pT3.

\section{CONCLUSIÓN}

La cirugía parcial renal laparoscópica no es una técnica sencilla, pero si oncológicamente efectiva. Debe realizarse por manos expertas, mimetizando los pasos que se utilizan en la cirugía abierta. Las indicaciones son las mismas para ambas técnicas. Se aconseja la oclusión vascular en masas renales mayores de $3 \mathrm{~cm}$ y con una profundidad mayor a $1 \mathrm{~cm}$ en el parénquima renal, para disminuir el sangrado y para disminuir al máximo el riesgo de márgenes positivos. La aparición de sustancias hemostáticas y sellantes pueden mejorar la hemostasia que constituye uno de los problemas de este tipo de técnica.

\section{REFERENCIAS}

1. Chow WH, Devesa SS, Warren JL, Fraumeni JF Jr. Rising incidence of renal cell cancer in the United Status. JAMA 1999;281(17):1628-1631.

2. Clayman RV, Kavoussi LR, Soper NJ, Dierks SM, Meretyk S, Darcy MD, et al. Laparoscopic nephrectomy: initial case report. J Urol. 1991;146(2):278-282.

3. Winfield HN, Donovan JF, Godet AS, Claymand RV. Laparoscopic partial nephrectomy: initial case report for benign disease. J Endourol. 1993;7(6):521-526

4. Gill IS, Matin SF, Desai MM, Kaouk JH, Steinberg A, Mascha E, Thornton J, Sherief MH, et al. Comparative analysis of laparoscopic versus open partial nephrectomy for renal tumors in 200 patients. J Urol. 2003;170(1):64-68.

5. Guillonneau B, Bermudez H, Gholami S, El Fettouh H, Gupta R, Adorno Rosa J, et al. Laparoscopic partial nephrectomy for renal tumor: single-center experience comparing clamping and no clamping techniques of the renal vasculature. J Urol. 2003;169(2):483-486.

6. Janetschek G, Abdelmaksoud A, Bagheri F, Al-Zahrani H, Leeb K, Gschwendtner M. Laparoscopic partial nephrectomy in cold ischemia: renal artery perfusion. J Urol. 2004; 171(1):68-71.

7. Rassweiler JJ, Abbou C, Janetschek G, Jeschke K. Laparoscopic partial nephrectomy: the European experience. Urol Clin North Am. 2000;27(4):721-736.

8. Ogan K, Cadeddu JA. Minimally invasive management of the small renal tumor: review of laparoscopic partial nephrectomy and ablative techniques. J Endourol. 2002,16(9): 635-643.

9. Gill IS, Desai MM, Kaouk JH, Meraney AM, Murphy DP, Sung GT, Novick AC.: Laparoscopic partial nephrectomy for renal tumor: duplicating open surgical techniques. $\mathrm{J}$ Urol. 2002;167(2Pt1):469-467.

10. Brown JA, Hubosky SG, Gomella LG, Strup SE. Handassisted laparoscopic partial nephrectomy for peripheral and central lesions: a review for 30 consecutive cases. J Urol. 2004;171(4):1443-1446.

11. Mendoza D, Newman RC, Albala D, Cohen MS, Tewari A, Lingeman $\mathrm{J}$, et al. Laparoscopic complications in markedly obese urologic patients (a multi-institutional review). Urology 1996;48(4):562-567.

12. Bermudez H, Guillonneau B, Gupta R, Adorno Rosa J, Cathelineau X, Fromont G, et al. Initial experience in laparoscopic partial nephrectomy for renal tumor with clamping of renal vessels. J Endourol 2003;17(6):373-378.

13. Bove P, Bhayani SB, Rha KH, Allaf ME, Jarrett TW, Kavoussi LR. Necessity of ureteral catheter during laparoscopic partial nephrectomy. J Urol. 2004;172(2):458460.

14. William K, Johnston III, MD, J. Stuart Wolf Jr, MD. Nefrectomía parcial laparoscópica: técnica, eficacia oncológica y seguridad Curr Urol Rep. 2005;4:69-77.

15. Jablonski P, Howden BO, Rae DA, Birrell CS, Marshall VC, Tange J. An experimental model for assessment of renal recovery from warm ischemia. Transplantation 1983;35(3): 198-204. 
16. Shekarriz B, Shah G, Upadhyay J: Impact of temporary hilar clamping during laparoscopic partial nephrectomy on postoperative renal function: a prospective study. J Urol. 2004;72(1):54-57.

17. Kane CJ, Mitchell JA, Meng MV, Anast J, Carroll PR, Stoller ML. Laparoscopic partial nephrectomy with temporary arterial occlusion: description of technique and renal functional outcomes. Urology 2004;63(2):241-246.

18. Rosales A, Salvador J, De Graeve N, Angerri O, Villavicencio H. Clamping of the artery in laparoscopic partial nephrectomy: an old device for a new technique. European Urology 2005;47(1):98-101.

19. Landman J, Venkatesh R, Lee D, Vanlangendonck R, Morissey K, Andriole GL, et al. Renal hypothermia achieved by retrograde endoscopic cold saline perfusion: technique and initial clinical application. Urology 2003;61(5): 1023-1025.

20. Gill IS, Abreu SC, Desai MM, Steinberg AP, Ramani AP, et al. Laparoscopic ice slush renal hypothermia for partial nephrectomy: the initial experience. J Urol. 2003;170(1): 52-56.
21. Wakabayashi Y, Narita M, Kim CJ, Kawakami T, Yoshiki T, Okada Y. Renal hypothermia using ice slush for retroperitoneal laparoscopic partial nephrectomy. J Urol. 2003;63 (4):773-775.

22. Polascik TJ, Meng MV, Epstein JI, Marshall FF. Intraoperative sonography for the evaluation and management of renal tumors: experience with 100 patients. J Urol. 1995; 154(5): 1676-1680.

23. Kubinsky DJ, Clark PE, Assimos DG, Hall MC. Utility of frozen section analysis of resection margins during partial nephrectomy. Urology 2004;64(1):31-34.

24. Hafez KS, Novisk AC, Campbell SC. Patterns of tumor recurrence and guidelines for followup after nephron sparing surgery for sporadic renal cell carcinoma. J Urol. 1997;157(6):2067-2070.

Dr. A. Rosales Bordes

Servicio de Urología. Fundació Puigvert

Cartagena 340-350 - 08025 Barcelona

E-mail: urologia@fundacio-puigvert.es 